\title{
Unusual clinical presentations of brucellosis in childhood
}

\author{
Authors \\ Mehmet Ulug ${ }^{1}$ \\ Ferda Yapici ${ }^{2}$ \\ Nuray Can-Ulug ${ }^{3}$ \\ ${ }^{1} \mathrm{MD}$, Özel Ümit Hospital, \\ Department of Infectious \\ Diseases and Clinic \\ Microbiology, Eskisehir, \\ Turkey \\ ${ }^{2} \mathrm{MD}$, Özel Sante Plus \\ Hospital, Department of \\ Pediatrics, Turkey \\ ${ }^{3} \mathrm{MD}$, Özel Ümit Hospital, \\ Department of Neurology, \\ Eskisehir, Turkey
}

Submitted on: 12/29/2010

Approved on: 12/29/2010

Correspondence to:

Mehmet Ulug

Özel Ümit Hastanesi,

Enfeksiyon Hastalıkları ve

Klinik Mikrobiyoloji

Kliniği

Batıkent Mah. Gerekli Sok.

No: 13, 26140 Eskișehir,

Turkey

mehmetulug21@yahoo.com

We declare no conflicts of interest.

(C)2011 Elsevier Editora Ltda. All rights reserved.
Dear Editor,

Brucellosis is a systemic infectious disease and it is still an important public health problem throughout the world, but especially in the Mediterranean region, including Turkey. The aim of this letter is to draw attention to atypical presentations of childhood brucellosis especially in endemic areas. A prospective study identified 112 consecutive patients with brucellosis admitted to our departments (Pediatrics and Infectious Diseases) between April 2007 and August 2008 at Midyat State Hospital, Mardin, Turkey. From these cases we present three patients with thrombocytopenia and maculopapular rash, pancytopenia and sternoclavicular arthritis, respectively.

\section{Case 1}

The 8-year-old girl was admitted with fever, weakness, nausea and epistaxis. Epistaxis had appeared for the last three days. She had a maculopapular rash particularly on her lower extremities. Complete blood count showed isolated thrombocytopenia of $23 \mathrm{~K} / \mathrm{UL}$ and increased median platelet volume (MPV) to $13 \mathrm{fl}$. The serum agglutination test (SAT) showed titers of $1 / 320$. Bone marrow aspiration revealed normal cellular distribution and maturation, but an increased number of megakaryocytes. Material for blood and bone marrow cultures were collected before the initiation antibiotic therapy [rifampicin and trimethoprimsulfamethoxazole (TMP-SMX) for six weeks]. Neither Brucella species nor other microorganisms were isolated from the cultures. The rash began to disappear within three days and thrombocytopenia began to resolve within four days of therapy commencement.

\section{Case 2}

An 8-year-old boy presented with a one-month history of weakness, sweats, and fever. On the day of admission, physical examination revealed that he was febrile and tachycardic.
Complete blood count at admission showed the following values: anemia ( $\mathrm{Hb}: 8.9 \mathrm{~g} / \mathrm{dL}$ ), leucopenia (WBC: $2600 / \mathrm{mm}^{3}$ ) and thrombocytopenia $(59,000 \mathrm{~K} / \mathrm{UL})$. The evaluation of bone marrow aspirate smears revealed hypercellularity, which was confirmed by the examination of bone marrow biopsy. Brucella spp. was isolated from bone marrow culture. On the $10^{\text {th }}$ day of the treatment (rifampicin and TMPSMX), the laboratory findings of the patient including pancytopenia completely resolved.

\section{Case 3}

A previously healthy 9 -year-old girl was admitted with a two-week history of fever, myalgias, chest and left shoulder pain. The sternoclavicular joint (SCJ) was tender on palpation, but there were no overlying erythema or edema, and there was severe limitation of movement of the left shoulder. The SAT showed titers of 1/640. X-ray appearances of chest and SCJ were normal, but computed tomography (CT) showed erosive changes of the medial end of the left clavicle and the articular surface of the manibrum. In the light of these findings, we thought of brucellar sternoclavicular arthritis and doxycycline plus rifampicin was started. After three days, the blood culture grew Brucella spp. She responded well to the treatment, her fever subsided three days after starting the therapy and pain in the shoulder gradually improved.

Brucellosis can affect people at any age, including children. Hematological alterations in brucellosis are common and resolve promptly with treatment. ${ }^{1}$ The hematological manifestations of brucellosis include anemia, leucopenia, neutropenia, thrombocytopenia and pancytopenia. ${ }^{2}$ Although the mechanism of the thrombocytopenia in brucellosis is not yet entirely known, it may be hypersplenism, bone marrow suppression due to septicemia, hemophagocytosis, disseminated intravascu- 
lar coagulation, granulomas and peripheral immune destruction of thrombocytes. ${ }^{2}$ In Case 1 , an increase in megakaryocytes in bone marrow and MPV in peripheral blood was thought by us that thrombocytopenia was related to immune destruction of thrombocyte in peripheral blood. The pathogenesis of pancytopenia in brucellosis has not been clearly understood, but it seems to be multifactorial as in thrombocytopenia. Bone marrow examination generally shows hypercellularity in patients with brucellosis and pancytopenia. ${ }^{3}$ We also detected hypercellularity on bone marrow examination in Case 2.

A variety of rarely-reported skin lesions has been described in brucellosis, including maculopapular lesions, papules, petechiae, purpura, impetiginous and psoriform lesions. ${ }^{4}$ The cutaneous findings observed in brucellosis are due to direct inoculation, hypersensitivity phenomena, deposition of immune complexes, and direct invasion by the organism reaching the skin hematogenously. ${ }^{4}$

The etiological agent in most cases of SCJ infection is Staphylococcus aureus. The other bacterial agents, especially Brucella spp., are less likely to be involved. ${ }^{5}$ Clinical presentation is often sudden, and patients might complain for days to months of atraumatic pain in the shoulder, neck or chest, with limited motion of the upper extremities, and associated fever. The diagnosis of the SCJ infection is therefore difficult. Routine radiographs are not helpful and CT scans may be of little help. In Case 3, the etiology documented by hemoculture, $\mathrm{x}$-ray of the SCJ was not helpful for diagnosis, and we documented a successful outcome of SCJ infection achieved only with medical treatment.

In conclusion, all physicians must recognize that brucellosis whether in an endemic region or not, remains a diagnostic puzzle due to occasional misleading unusual presentations and non-specific symptoms.

\section{REFERENCES}

1. Tanir G, Tufekci SB, Tuygun N. Presentation, complications, and treatment outcome of brucellosis in Turkish children. Pediatr Int 2009; 51:114-9.

2. Dilek I, Durmus A, Karahocagil MK et al. Hematological complications in 787 cases of acute brucellosis in eastern Turkey. Turk J Med Sci 2008; 38:421-4.

3. Karakukcu M, Patiroglu T, Ozdemir MA et al. Pancytopenia, a rare hematologic manifestation of brucellosis in children. J Pediatr Hematol Oncol 2004; 26:803-6.

4. Metin A, Akdeniz H, Buzgan T et al. Cutaneous findings encountered in brucellosis and review of the literature. Int J Dermatol 2001; 40:434-8.

5. Gillis S, Friedman B, Caraco Y et al. Septic arthritis of the sternoclavicular joint in healthy adults. J Intern Med 1990; 228:275-8. 